\title{
Chronic Empyema: Aetiopathology and Management Challenges in the Developing World
}

\author{
Martins Oluwafemi Thomas ${ }^{1,2 *}$, Ezekiel Olla Ogunleye ${ }^{1,2}$ \\ ${ }^{1}$ Lagos University Teaching Hospital, Surulere, Lagos, Nigeria \\ ${ }^{2}$ College of Medicine, University of Lagos, Surulere, Lagos, Nigeria \\ E-mail: *oluwafemithomas@yahoo.com \\ Received July 4, 2011; revised September 21, 2011; accepted October 13, 2011
}

\begin{abstract}
Objectives: Chronic empyema thoracis (CET) is common worldwide despite widespread use of highly effective antibiotics. Also, newer technological armamentaria are available for its diagnosis and treatment. This research was performed to study the aetiopathological profile and the management challenges of CET in view of the background information stated above. Methods: It is a prospective study spanning a period of 62 months in Lagos, Nigeria. Patients' bio-data, aetiological factors, salient clinical features, management modalities and complications of care were documented and analysed. We excluded patients who were not fit for surgery and those who could not afford surgery. Patients that were initially registered as chronic empyema patients but who later became positive for malignancy were also excluded. Results: There were 93 patients (61 males and 31 females). Age range was 4 - 72 years but the range 20 - 49 years constituted $71.0 \%$ of the studied population. Poorly treated acute chest infections was the aetiological factor in $49.5 \%$ of patients. Tuberculosis was established in 37 patients (39.8\%) who were especially in social classes I and II. Decortication and pneumonectomy were done for $52.7 \%$ and $16.1 \%$ of the patients respectively. Average hospitalisation was prolonged for pneumonectomy because some patients had pneumonectomy space infections. Discusion: CET remains a worldwide problem despite widespread use of potent antibiotics. There are newer diagnostic and therapeutic armamentaria that are not readily available in developing world thereby posing major challenges to practicing surgeons.
\end{abstract}

Keywords: Chronic Empyema Aetiopathology Management

\section{Introduction}

Empyema thoracis (ET) is collection of pus in pleural cavity and it is common worldwide. Despite availability and widespread use of highly effective antibiotics, empyema thoracis occurs and is associated with significant morbidity and occasional mortality [1]. Globally, respiratory diseases are a leading cause of morbidity and mortality among both children and adults.

ET affects both sexes and no age is exempted. The clinical and pathological features vary with duration of ailment. In the early phase, the features are those of acute respiratory infection, characterised by cough, fever and ipsilateral chest pain. In the late phase, fever, is less pronounced. Features like worsening dyspnoea, weight loss and cough supervene.

Various aetiological factors of empyema thoracis have been identified. Parapneumonic empyema continues to be a disease of significant morbidity and mortality among children despite recent advances in medical management [2].

Streptococcus pneumonia is a major respiratory pathogen and the spectrum of clinical presentations highly associated with this pathogen include bacteraemic and non-bacteraemic presentations of pneumonia as well as parapneumonic effusions or empyema [2].

In developing countries, antecedent conditions such as malnutrition, measles or infection with antibiotic-resistant organisms may increase the risk of severe pneumonia accompanied by empyema. Other co-morbid conditions may include diabetes mellitus and HIV/AIDS. Many countries of the world have adopted the use of pneumococcal vaccine in children. This has been found effective in reducing the risk of pneumococcal infec- 
tions.

Trauma is another important cause of empyema thoracis. Haemothorax, pneumothorax or haemo-pneumothorax can be secondarily infected leading to ET. Attempt at treatment, if not handled professionally, can also lead to ET. Pleural effusions (exudative or transudative) can proceed to frank empyema thoracis if not properly managed. In all the conditions mentioned above, early institution of definitive care will stop the progression of acute ET to chronic empyema. However, delay in termination of the progression often leads to formation of fibrinous strands thereby laying the foundation for fibrous adhesions, loculation of empyma and formation of a thick fibrous cortex as in the complex of chronic empyema thoracis (CET).

CET is therefore an important sequela of untreated or poorly treated empyema thoracis. The bacteriology of chronic empyema is fairly diverse [1,3-5]. A study of empyema thoracis in India revealed that aetiology was tubercular in $42 \%$ of patients whereas, the rest were bacterial. Among the bacterial cases, they found Staphylococcus aureus as the commonest organism implicated [1]. In the same study, the rest of the patients had gram negative infection by Pseudomonas aerugenosa, Klebsiella pneumoniae and Escherichia coli. While these organisms can occur singly, mixed infections are possible and a good number may be culture negative. Outside the regular causes of chronic empyema stated (vide supra), it is important to note the rare causes of chronic empyema like achalasia [6].

The spectrum of ensuing pathology of chronic empyema is fairly wide. It may range from small encysted empyema (which may occur singly or in multiples), through varying degrees of encasement of lobes or whole lung, and up to varying degrees of parenchymal destructions involving lung segments, lobes or whole lung.

Destroyed lung is now accepted to designate the large destructions of the lung, secondary to pulmonary and essentially infectious diseases, the cure of which is obtainable but with possible important sequelae [7].

This spectrum of pathological entities constitute the indications for surgical intervention in chronic empyema. It is also important to know that failure of prolonged medical treatment, multi-drug resistance of tubercle bacilli and continuing haemoptysis constitute important indications for surgery. Either of the lungs or both can be affected. There is no agreement on the dominance of one lung over the other when it comes to frequency of occurrence $[1,8]$.

The diagnostic armamentarium is fairly diverse. Chest radiographs complimented with computerised tomographic scan of the chest are often sufficient to study anatomic details of chronic empyema. Microbiology is of essence in determining the organisms present in the empyema and their sensitivity to known antimicrobials.

The surgical treatment modalities of chronic empyema are dependent on the targeted pathologic entity. Rib resection and open drainage had been in use for a long time. Decortication is done to peel the fibrous cortex that encases the lung. When there is parenchymal damage, varying degrees of lung resection are indicated depending on the extent of the damage. Rarely, this may be multi-segmentectomy, but more commonly lobectomies, bi-lobectomies or pneumonectomies are often necessary. All these are now being done by Video Assisted Thoracoscopic Surgery (VATS). Elective postoperative ventilation is often indicated. Common complications of lung resections include cardiac arrest, pneumonectomy space infection, bronchopleural fistula formation amongst others.

This study was conducted to determine the aetiopathology and management challenges of chronic empyema in a developing world. It is actually a research to see the aetiopathological profile and management challenges of CET in view of widespread use of antibiotics and available modern armamentaria for its management. It is expected to further improve the current knowledge and understanding of the various aspects of chronic empyema.

\section{Methods}

The study was conducted at the Lagos University Teaching Hospital in southwest Nigeria from October 1999 to December 2005.

In a prospective setting, data sheets were designed to accommodate the needed parameters. The parameters were recorded for patients who were referred to our team in Lagos metropolis with diagnosis of chronic empyema thoracis. We recorded their bio-data with a particular note of ages, sexes and social classes.

We noted the followings:

- Duration of their ailment;

- Clinical evidence of cardiac involvement;

- The lung or lungs affected;

- Aetiological factors that were found in them;

- Method(s) of diagnosis implored.

Diagnoses were premised on clinical features and laboratory reports.

We specifically sought the following features:

- History of trauma;

- Previous diagnosis of pulmonary tuberculosis;

- Previous diagnosis of acute chest infection;

- History of any chest procedures like needle aspiration;

- Previous chest operation like tube thoracostomy and thoracotomy. 
We also noted the outcomes of laboratory diagnoses like:

- Radiographs and computerised axial tomograph of chest;

- Microbiology including mantoux test, bacteriology, and mycology of aspirates;

- cytology of chest aspirates.

The final treatment modalities were recorded for each of them. Specifically, we recorded the definite operations performed like decortication, lobectomy, pneumonectomy. Postoperative complications were also noted for each patient when they occurred. The data sheets were studied and analysed manually. We calculated measurements for dispersion of the distribution.

We excluded patients who were not fit for surgery and those who could not afford surgery because we thought they would not be available to complete the research. Patients that were initially registered as chronic empyema patients but who later became positive for malignancy were excluded.

We also excluded rib resection drainage because the few patients involved eventually underwent definitive operations. The main issue here was that it became increasingly difficult to manage their effluents because of the rising cost of drainage bags.

We noted the duration of hospitalisation and operation related deaths.

\section{Results}

The study period lasted 62 months and 93 patients were involved. They were 61 males and 32 females giving an M:F ratio of 1:1.9 (Table 1). The age range was $4-72$ years but age range $20-49$ constituted $71.0 \%$ of all the patients. Within the latter age range, 46 patients (69.7\%) were males. Further analysis showed no significant differences in sex distribution at the extremes of age.

Poorly treated acute chest infection was the aetiological factor in $49.5 \%$ of patients. (Table 2) Tuberculous aetiology was established in 37 patients (39.8\%) and $83.8 \%$ of them occurred in patients of social classes I and II (Table 2). In all patients in social classes I and II constituted $79.6 \%$ of the studied population, none of the patients was in social class $\mathrm{V}$.

Decortication was done for 49 patients constituting $52.7 \%$ while pneumonectomy was done for $16.1 \%$ of the patients. (Table 3) Average hospitalisation for decortication was 16.7 days while lobectomy and pneumonectomy patients spent 18.2 and 24.1 respectively. Average hospitalisation was prolonged for pneumonectomy because of the weight effect of 3 patients who had pneumonectomy space infections and they all had prolonged hospitalisation.
Table 1. Age and sex distribution of patients.

\begin{tabular}{|c|c|c|c|}
\hline Age (yr) & $\mathbf{M}$ & $\mathbf{F}$ & Total \\
\hline$<10$ & 1 & - & $1(1.1 \%)$ \\
\hline $10-19$ & 1 & 1 & $2(2.2 \%)$ \\
\hline $20-29$ & 13 & 8 & 21 (22.6\%) \\
\hline $30-39$ & 16 & 8 & 24 (25.8\%) \\
\hline $40-49$ & 17 & 4 & 21 (22.6\%) \\
\hline $50-59$ & 8 & 5 & 13 (13.9\%) \\
\hline \multirow[t]{2}{*}{60 and above } & 5 & 6 & 11 (11.8\%) \\
\hline & $61(65.6 \%)$ & $32(34.4 \%)$ & $93(100.0 \%)$ \\
\hline
\end{tabular}

Table 2. Aetiological factors and social classes.

\begin{tabular}{ccccccc}
\hline Aetiological & \multicolumn{7}{c}{ Social class } \\
\hline Factors & I & II & III & IV & V & Total \\
\hline Chest trauma & 1 & 2 & - & - & - & 3 \\
& & & & & & $(3.2 \%)$ \\
Tuberculosis & 18 & 13 & 6 & - & - & $\begin{array}{c}37 \\
(39.8 \%)\end{array}$ \\
& & & & & & 46 \\
Poorly treated & 14 & 19 & 8 & 5 & - & $(49.5 \%)$ \\
& & & & & - & 7 \\
Iatrogenic & 5 & 2 & - & - & - & $(7.5 \%)$ \\
Pneumonia & 38 & 36 & 14 & 5 & - & 93 \\
& $(40.9 \%)$ & $(38.7 \%)$ & $(15.1 \%)$ & $(5.4 \%)$ & $(0.0 \%)$ & $(100.0 \%)$ \\
& & & & & &
\end{tabular}

Table 3. Treatment modalities.

\begin{tabular}{cc}
\hline Modality & No of patients \\
\hline Decortication & $49(52.7 \%)$ \\
Lobectomy & $29(31.2 \%)$ \\
Pneumonectomy & $15(16.1 \%)$ \\
Total & $\mathbf{9 3 ( 1 0 0 . 0 \% )}$ \\
\hline
\end{tabular}

There were three operative deaths. One death followed decortication while the other 2 were for lobectomies. Therefore operative mortalities were $0.0 \%$ for pneumonectomy, $2.0 \%$ for decortications and $3.4 \%$ for lobectomies.

Based on the exclusion criteria stated (vide supra), 28 other patients who got enlisted initially were dropped from the study.

\section{Discussion}

Empyema thoracis remains a common problem world- 
wide. Its pathological spectrum range from acute, through the sub-acute to chronic states. These three phases are also described as types I, II and III respectively. The acute and sub-acute forms are easily managed with chest tube insertion and antibiotics most of the times. Management of the chronic form is more challenging both technically and in terms of costs to the patients especially in a developing world. Technological advancement has improved the diagnostic and therapeutic armamentaria of chronic empyema but these have not lessened the financial burden. In our study, we chose to look at the aetiopathological profile and the technical challenges of managing empyema thoracis with particular interest in the developing world.

Using our study criteria which are fairly strict, 93 patients in the chronic empyema bracket a in a 62-month period is a major haul and this exposes the burden of the disease in the developing world. It therefore comes to reason that this is a major problem with its attendant technical and financial challenges. The $65.6 \%$ preponderance of males over females as in this study, is fairly consistent with earlier researches in Taiwan, China, South Korea Vietnam, India and Nigeria [1,2,4-6]. We believe that this is the cumulative effect of the fact that men are generally more predisposed to the aetiological factors of chronic empyema. The predominant age range of $20-49(71.0 \%)$ is also consistent with earlier Asian and African studies [1,2,4-6].

The aetiology of chronic empyema as found from this study, though fairly diverse, is largely dominated by poorly treated pneumonias (49.5\%) and pulmonary tuberculosis (39.8\%). This is of major concern in view of the growing influence of HIV/AIDS on the epidemiology and pathology of tuberculous infections. Another point of concern in the distribution of the studied population is the occurrence of $79.6 \%$ of patients in social classes I and II. This constitutes a major financial burden for this group of people especially where there is no health insurance programme.

From our study, decortication was more commonly indicated than pneumonectomy. As demonstrated in Table $3,52.7 \%$ of our patients had decortication while $16.1 \%$ underwent pneumonectomy. We had our challenges in the postoperative care of patients who had rib resection drainage procedures because of the rising cost and relative non-availability of drainage bags. This limited the number of such procedures in our study.

Average hospitalisation for decortication was 16.7 days while lobectomy and pneumonectomy patients spent 18.2 and 24.1 respectively. These hospitalisation figures are generally shorter when compared with the patients treated with fibrinolytic agent in India [1]. We also noted the role of pneumonectomy space infection in prolonging hospitalisation. This can be reduced with more strict preoperative and intra-operative care.

Another challenge is that Video Assisted Thoracoscopic surgery (VATS) which is a proven method of reducing hospitalisation is not readily available in the developing world. Therefore patients who ordinarily should benefit from this technology are deprived of such benefit on the basis of non-availability.

The overall operative mortality was $3.2 \%$. A breakdown revealed zero mortality for pneumonectomy, $2.0 \%$ for decortications and $3.4 \%$ for lobectomies. This is the trend in many of the previous studies in Asia and Africa $[1,2,6]$.

We concluded that there is no significant change in aetiology of chronic empyema over the years and that the spectrum of its pathology is also constant. However, the developing world is not catching up with the evolving technological advancement in the management of chronic empyema. This, therefore, is a major challenge for thoracic surgeons and their patients.

We therefore recommend that practitioners in the developing world should acquire the needed expertise and procure VATS equipment to reduce postoperative morbidity of empyema surgery and halt the progression to chronic empyema.

\section{Acknowledgements}

I wish to acknowledge the secretarial support of my dear daughter Oyindamola THOMAS. She assisted greatly in typing the manuscript.

\section{References}

[1] A. Banga, G. C. Khilnani, S. K. Sharma, A. B. Dey, W. Naveet and B. Namrata, "A Study of Empyema Thoracis and Role of Intrapleural Streptokinase in Its Management,” BMC Infectious Diseases, Vol. 4, No. 6, 2004, pp. 1-7.

[2] B. Nyambat, P. Kilgore, D. Yong, D. Anh, C. Chiu, X. Shen, L. Jodar, T. Ng, H. Bock and W. Hansdorff, "Survey of Childhood Empyema in Asia; Implication for Detecting the Unmeasured Burden of Culture-Negative Bacterial Disease,” BMC Infectious Diseases, Vol. 8, No. 8, 2008, pp. 8-15.

[3] S. A. Adebonojo, O. Adebo and O. Osinowo, "Pattern of Thoracic Surgical Diseases in Nigeria: Experience at the University College Hospital, Ibadan," Journal of the National Medical Association, Vol. 70, No. 9, 1978, pp. 651-657.

[4] I. A. Grillo and S. Bohrer, "Surgical Management of Intrathoracic Tuberculosis in Ibadan, Nigeria," West African Medical Journal and Nigerian Practitioner, Vol. 20, No. 4, 1971, pp. 269-273.

[5] S. A. Adebonojo, O. Osinowo and O. A. Adebo, "Pneu- 
monectomy in Nigeria: Indications and Results,” Journal of the National Medical Association, Vol. 71, No. 11, 1979, pp. 1077-1080.

[6] N. Mehdi, M. Weinberger and M. Abu-Hasan, “Achalasia: Unusual Cause of Chronic Cough in Children,” Cough, Vol. 4, No. 6, 2008, pp. 16-21.

[7] M. F. Carette, F. Blanchon, B. Milleron and H. Brocard,
“Destroyed Lung,” Semaine des Hopitaux, Vol. 55, No. 17-18, 1979, pp. 843-853.

[8] S. A. Adebonojo, O. A. Adebo, O. Osinowo and I. A. Grillo, "Management of Tuberculous Destroyed Lung in Nigeria," Journal of the National Medical Association, Vol. 73, No. 1, 1981, pp. 39-42. 